\title{
Integration of Semiconductor Nanowire Lasers with \\ Polymeric Waveguide Devices on a Mechanically
}

\section{Flexible Substrate}

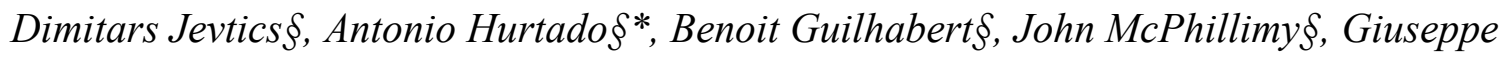

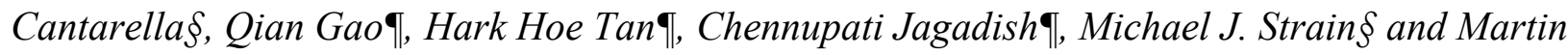 \\ D. Dawson§
}

\begin{abstract}
§Institute of Photonics, SUPA Department of Physics, University of Strathclyde, Technology and Innovation Centre, 99 George Street, G1 1RD, Glasgow, United Kingdom.

ףDepartment of Electronic Materials Engineering, Research School of Physics and Engineering, The Australian National University, Canberra, ACT2601, Australia.
\end{abstract}

KEYWORDS. Nanowire Lasers, Nanowire Coupling, Photonic Integration, Nanophotonics 
ABSTRACT. Nanowire lasers are integrated with planar waveguide devices using a high positional accuracy micro-transfer printing technique. Direct nanowire to waveguide coupling is demonstrated, with coupling losses as low as $-17 \mathrm{~dB}$, dominated by mode mismatch between the structures. Coupling is achieved using both end-fire coupling into a waveguide facet, and from nanowire lasers printed directly onto the top surface of the waveguide. In-waveguide peak powers up to $11.8 \mu \mathrm{W}$ are demonstrated. Basic photonic integrated circuit functions such as power splitting and wavelength multiplexing are presented. Finally, devices are fabricated on a mechanically flexible substrate to demonstrate robust coupling between the on-chip laser source and waveguides under significant deformation of the system.

The integration of micron scale laser sources with on-chip photonic integrated circuits (PICs) is of great interest for applications from low-power, short haul optical interconnects [1] to point of use biosensor systems [2]. By locating laser sources directly on-chip, the critical alignment of fiber to waveguide couplers can be overcome and sources can be distributed arbitrarily across the chip, reducing packaging costs for volume manufacture of such devices. Furthermore, recent advances in the manufacture of planar optics on flexible substrates have demonstrated the potential for high performance deformable devices in applications including mechanical sensors [3], optical interconnect fabrication [4] and flexible silicon photonics [5]. Such systems are critically dependent on the availability of on-chip optical sources for point of use application.

There are a number of potential schemes for the hybrid integration of laser sources with passive planar optics, including die bonding [6] and pick-and-place [7] technologies. However, these schemes tend to work with modestly sized edge-emitting laser devices with lengths in the 10's to 
100 's of microns range. An alternative approach lies in the use of nanowire (NW) semiconductor laser devices. These sources have dimensions in the micron range and have been demonstrated in optically [8][9] and electrically [10] pumped variants, with average output powers in the nW regime [11]. There are two main methods for the integration of NWs with on-chip optical waveguides: direct growth of semiconductor NWs on the passive waveguide material [12, 13], or mechanical transfer of NWs from their growth substrate to the target devices $[14,15,16]$. Regrowth methods allow high accuracy alignment between physical structures on different material platforms through lithographic methods and have been reported with coupling efficiencies between NW array lasers and silicon waveguides of $\sim 4 \%$. Mechanical transfer techniques avoid subjecting the host devices to growth process conditions and allow the prescreening of individual devices to be integrated. The crucial issue in the integration of these laser sources with PICs lies in the coupling of their optical output mode with the on-chip optics. A number of techniques for the mechanical positioning and organization of NWs have been reported in recent years, such as optical and optoelectronic tweezing [17], dry transfer techniques [18], mechanical pick-up using atomic force microscopy tips [19], fluidic [20] and electric field processes [21], Langmiur-Blodgett assembly protocols [22] and large scale transfer printing processes [23-26]. In all of these techniques there is a major tradeoff between the final NW placement accuracy and the potential scalability of the method. It has therefore been necessary to employ self-aligned coupling schemes to couple NW laser modes to guided wave structures, for example (with reported external coupling efficiencies given in parentheses), v-groove plasmonic guides (10\%) [27], air-slot photonic crystals cavities [14] or lateral mechanical contacting of NW sections coupled to long nano-ribbon waveguides (50\%) [28]. In addition to coupling efficiency of the NW output mode to external guiding structures, the form of and fabrication method of 
such waveguides is important to consider for any given application, taking into account, for example, propagation losses, effects on NW threshold, mode geometry, and potential integration with lithographically defined photonic integrated circuits. Finally, future integration of these NWs will require electrical injection for many applications. Pioneering work has already shown the compatibility of mechanically placed NW devices with electrical injection [10, 20, 21].

The requirement of scalable, high precision placement of NW sources can be met using a nanoscale Transfer-Printing (nano-TP) method [29]. In this work we demonstrate, by means of this technique, the integration of NW laser sources with pre-fabricated PICs. Two different coupling geometries are presented, printing the NW laser either on top of existing waveguides or directly aligned with the waveguide facet. Multiple NWs are integrated with power splitters and on single waveguides to show multi-wavelength operation. Finally, the integration of NW lasers with PICs on a flexible glass substrate is realized, demonstrating consistent laser-waveguide coupling under severe deformation of the substrate.

In order to directly couple NW lasers with planar waveguide devices, two geometries were investigated, as shown in the schematic of Figure 1a: (i) lateral coupling and (ii) end-to-end facet coupling. In (i), the NW laser is directly printed onto the top surface of the waveguide. In (ii), the NW laser is printed onto the substrate with its long axis aligned with the normal of the waveguide end facet. InP NWs were employed in this work, with lasing emission at room temperature in the range of $\sim 840-900 \mathrm{~nm}[30][31]$, though the technique is equally applicable to NWs emitting in other spectral ranges. The NW lasers were hexagonal in cross-section with an equivalent diameter of $435 \mathrm{~nm}$ and an average length of $6 \mu \mathrm{m}$. The waveguide devices were fabricated on $\mathrm{SiO}_{2}$ substrates in SU-8 polymer material using a custom direct-write laser lithography tool (see Supporting Information). The waveguides were $7 \mu \mathrm{m}$ in width and $4 \mu \mathrm{m}$ in 
height and are therefore multimode at the wavelengths of interest. Multimode waveguides allow for an increased numerical aperture (NA) for input coupling and are compatible with high bandwidth, short haul optical interconnect techniques [32]. Propagation loss of the waveguides is estimated to be less than $3 \mathrm{dBcm}^{-1}$ (see supplementary material for details). Finally, the NW lasers were integrated with the waveguide platform by means of a nano-TP method [29]. This technique provides high precision placement accuracy [33] thus allowing the selection of pretested NW lasers for integration with the waveguides. The accuracy with which the NWs could be aligned with pre-existing features on the host substrate was assessed by printing pairs of NWs in an end-to-end configuration, attempting to align their long axes. Full details of this measurement are given in the supporting information document. Figure 1b shows an SEM image of two NWs printed in this configuration. The average misalignment of pairs of NWs printed in this form was measured to be $50 \mathrm{~nm}$ with a standard deviation of $35 \mathrm{~nm}$, illustrating the capability of this method for alignment of NW devices to optical waveguides with crosssectional dimensions down to the sub-micron range.

Figure 1c shows a typical lasing spectrum and an image of the emission from a NW laser obtained using the $\mu$-photoluminescence ( $\mu$-PL) setup (shown in figure 1d) built to characterize the room-temperature lasing emission of the integrated NW lasers. These were optically pumped with a $532 \mathrm{~nm}, 1.6 \mathrm{~ns}$ pulsed frequency-doubled Nd:YAG laser at a repetition rate of $10 \mathrm{kHz}$. All laser results presented in this work were measured at an average optical pump power of $6.95 \mu \mathrm{W}$ (fluence: $38.8 \mathrm{~mJ} / \mathrm{cm}^{2}$ ). The measurement rig consisted of two main sub-sections, a vertical detection setup (VS) for measurement of the top scattered light from the NW lasers, and an edge detection module (EDM), where light from the waveguide end facets was imaged on a CCD 
camera. Full details of the setup are given in the supplementary material. The results in figure 1c were captured with the VS detection system.

Figure 2 shows results demonstrating the successful integration of individual InP NW lasers with SU-8 waveguides in both lateral and facet coupling configurations (see Figures 2a-c). The micrographs in Figure $2 \mathrm{~d}$ and $2 \mathrm{e}$ show the lasing emission from the NWs in Figure $2 \mathrm{~b}$ and $2 \mathrm{c}$ (as collected with the VS detection module) after their integration with the waveguides. The light coupled into the waveguides was imaged at the waveguide's facet (approx. $1 \mathrm{~cm}$ away from the NW lasers' location) using the EDM system in the setup. These images are shown in Figure $2 f$ and $2 \mathrm{~g}$, for the lateral and facet configurations, respectively. Additionally, Figures $2 \mathrm{~h}$ and $2 \mathrm{i}$ plot the lasing spectra measured (using the VS detection modules) for the NW lasers in Figures $2 b$ and $2 c$, respectively. Figures $2 d-i$, therefore illustrate that the NWs retain their optical properties [29] after their integration with the waveguides and that a fraction of their light is successfully coupled into the waveguides. Figures $2 \mathrm{f}$ and $2 \mathrm{~g}$ also reveal a complex multimode spatial profile as the NW laser emission couples into different modes of the multimode polymer waveguide.

The coupling efficiencies for both test geometries can be calculated using the integrated power measured at the EDM's camera. This signal is then compared with measurements of a set of similar NW lasers printed at the edge of a substrate and imaged directly. For the latter measurements, a number of NWs were printed at the edge of a cleaved $\mathrm{SiO}_{2}$ substrate. These permitted the direct imaging of their end-facet emission thus allowing the calculation of their emitted optical powers. Full details on the coupling efficiency calculations are given in the supporting information document. The calibration NW lasers were optically excited with a fluence of $38.8 \mathrm{~mJ} / \mathrm{cm}^{2}(6.95 \mu \mathrm{W})$, (with the pump laser spot being much larger than the crosssectional absorption area of the NWs). Under these conditions these were measured to lase with 
an average output optical power of $11.2 \mathrm{nW}$ (as collected at the CCD camera of the EDM system), corresponding to a peak power of $700 \mu \mathrm{W}$. In parallel, the measured integrated average powers of the waveguide modes for the lateral and facet coupled devices were $0.07 \mathrm{nW}$, and 0.19 $\mathrm{nW}$, respectively. These give values of peak power from the waveguides equal to $4.2 \mu \mathrm{W}$ and 11.8 $\mu \mathrm{W}$, respectively. When compared with the calibration devices, this yields coupling efficiencies of $0.7 \%(-21.5 \mathrm{~dB})$ and $1.9 \%(-17.2 \mathrm{~dB})$ for the lateral and facet coupled NW lasers, respectively.

To demonstrate the flexibility and simplicity of the method, a number of other photonic integrated circuit (PIC) components were created. The first of these was a Y-junction power splitter. Here, two NW lasers were integrated in facet coupled arrangement at the two symmetric ports of the splitter and the optical waveguide mode was measured at the single waveguide output as seen in Figures 3 a-c. Total propagation length from the NW lasers to the output facets was around $1 \mathrm{~cm}$. Figures $3 \mathrm{~d}$ and $3 \mathrm{e}$ show the facet images captured at the single output waveguide facet of the $2 x 1$ device when the NW lasers in figs. $3 b$ and $3 c$ are individually (optically) pumped above their lasing thresholds. The waveguide facet images revealed a relative integrated power difference of only $9 \%$ between the NW lasers integrated at the top and bottom branches of the Y-junction (with the latter having higher power). This illustrates consistent coupling across different waveguides and NW lasers. We attribute this difference in detected powers to asymmetries in the Y-junction and possible misalignments between the former and the NWs. Additionally, some asymmetry in the waveguide junction can be observed from the variation of the output waveguide modes from each injection NW laser. For example, Figure $3 \mathrm{~d}$ shows that the fundamental mode is dominantly coupled to from the top waveguide arm, whilst Figure $3 \mathrm{e}$ shows a significant amount of optical power transmitted in the first order mode, 
injected from the lower arm. These variations are likely due to asymmetry in the waveguide pattern from the laser writing tool in the 10's of nanometers scale and variable offset of the NW lasers from the waveguide center, inducing different coupling fractions to the allowed guided modes.

Using the lateral coupled NW laser configuration, an alternative method for producing multiplexed signals in a single waveguide can be created. Figure 4a shows an optical micrograph of a waveguide with two NW lasers printed along its length, separated by $\sim 50 \mu \mathrm{m}$. This geometry has the potential for integrating many sources together in an extremely compact footprint. The measured lasing emission, captured in the VS, is shown in Figure $4 \mathrm{~b}$ and $4 \mathrm{c}$. The light imaged at the waveguide facets is shown in Figure $4 \mathrm{~d}$ and $4 \mathrm{e}$. Estimations of the integrated powers revealed a difference in the values obtained for both NW lasers. Finally, Figure $4 \mathrm{f}$ merges the individually measured spectra (at the waveguide's facet) for both integrated NW lasers, showing achievement of two distinct lasing peaks at different wavelengths, at 861 and 882 $\mathrm{nm}$, for the NWs integrated on a single waveguide.

Finally, one of the major benefits in integrating NW laser sources on-chip with planar waveguides is the reduction in alignment accuracy necessary to couple to external systems. Usually, on-chip waveguides have to be aligned with sub-micron precision to injection fiber or free space optics. In systems based on flexible substrates this makes operation of on-chip devices extremely challenging. In the scheme presented here, the NW laser is aligned with the waveguide devices using the nano-TP method. Therefore, under deformation of a flexible substrate, the coupling between the on-chip elements should not be significantly affected. The NW lasers still require to be optically pumped, but this alignment is far more tolerant to 
misalignment than coupling to waveguides, as the pump spot can be much larger than the NW laser geometric cross-section, allowing for coarse alignment.

The integrated optical devices presented in this work were fabricated on a flexible glass substrate with a thickness of $30 \mu \mathrm{m}$ [34]. To demonstrate the robustness of the NW laser to waveguide coupling under mechanical deformation of the system, the setup shown in Figure 4a was used. The flexible glass substrate was mounted with the output facet end bonded to the edge of a glass slide to keep it fixed in position. The free end of the substrate was deflected in the vertical direction using a mechanical probe tip on a micrometric translation stage. This deflection was converted to an equivalent radius of curvature, $R_{c}$, assuming a constant bend radius of the flexible substrate. The NW laser was integrated with the waveguide in a facet configuration as shown in Figure 5b. Figure 5c shows the VS image of the NW laser under pumping whilst Figure $5 \mathrm{~d}$ shows the imaged facet of the waveguide. The integrated waveguide facet power was measured as a function of $1 / R_{c}$, where $1 / R_{c}=0$ corresponds to an un-deflected substrate. Figure $5 \mathrm{e}$ shows the relationship between the substrate bending and normalized output power of the waveguide. At each point the pump laser was optimized to produce a maximum measured power, i.e. compensating for vertical misalignment of the pump focus with deflection of the substrate. There is minimal variation $(\sim 5 \%)$ in the measured output power, and therefore the coupling efficiency between the NW laser and the waveguide, down to a radius of curvature of $1.6 \mathrm{~cm}$. This was the limit of our measurements due to mechanical fracture of the substrate for radius below $1.6 \mathrm{~cm}$.

In summary, this work presents the hybrid integration of NW lasers with polymeric waveguide devices on mechanically flexible substrates, including channel waveguides, Y-junctions and multi-wavelength systems. Two integration configurations (facet and lateral coupling) are 
successfully demonstrated, offering alternative routes to on-chip device design. Coupling efficiency estimations showed that optical power levels in the order of several $\mu \mathrm{Ws}$ (peak power) can be coupled from the integrated NW lasers into the waveguides and propagated over significant on-chip distances. The coupling efficiencies for facet and lateral coupling of $-17 \mathrm{~dB}$ and $-21 \mathrm{~dB}$, respectively, are dominated by the spatial mode mismatch between the NW lasers and polymer waveguides. This modal mismatch can be improved using optimized modematching couplers, for example tapered waveguide designs [35] or embedding the emitter within the waveguide structure [36]. Finally, the $\mu$-assembled laser to waveguide coupling overcomes key limitations of PICs on flexible substrates. Coupling efficiency is robust against mechanical substrate deformation, requiring only coarse alignment of the pump laser spot. Future systems, integrating vertical pump diodes, NW lasers and PICs enabled by this scheme are particularly relevant for optical interconnects and point of use biosensors on flexible substrates.

\title{
ASSOCIATED CONTENT
}

Supporting Information. Additional information on the nanoscale Transfer Printing technique, waveguide fabrication, $\mu$-Photoluminescence setup for the characterization of NWs, positional accuracy of the printing method, and estimation of the NW-waveguide coupling efficiency. The supporting information is available free of charge on the ACS Publications website at DOI: $\mathrm{xxxxx/acs.nanolett.xxxxxxx.}$

\author{
AUTHOR INFORMATION
}

Corresponding Author 


\author{
*Antonio Hurtado, Institute of Photonics, University of Strathclyde, Technology and Innovation \\ Centre, 99 George Street, G1 1RD, Glasgow (United Kingdom), E-mail: \\ antonio.hurtado@strath.ac.uk
}

\begin{abstract}
Author Contributions
The manuscript was written through contributions of all authors. All authors have given approval to the final version of the manuscript. The team at the University of Strathclyde carried out the nanoscale Transfer Printing, waveguide fabrication and device characterization experiments. The team at the Australian National University fabricated the InP NW lasers. All authors contributed to the writing of the manuscript.
\end{abstract}

\title{
Funding Sources
}

The Australian authors would like to acknowledge funding from the Australian Research Council. The Australian National Fabrication Facility established under the Australian Government's National Collaborative Research Infrastructure Strategy, is gratefully acknowledged for providing access to some of the facilities used in this work.

\section{Notes}

The authors declare no competing financial interest.

\section{REFERENCES}

[1] Miller, D. A. B., J. Lightw. Technol. 2017, 35, 346-396. 
[2] Ruoxue, Y, Park, J.-H., Choi, Y., Heo, C.-J., Yang, S.-M., Lee, L.P., and Yang, P., Nat. Nanotech. 2012, 7, 191-196.

[3] Bhola, B., Song, H., Tazawa, H., and Steier, W., IEEE Photon. Technol. Letts., 2005, 17, 867-869.

[4] Bosman, E., Van Steenberge, G., Van Hoe, B., Missinne, J., Vanfleteren, J., and Van Daele, P., IEEE Photon. Technol. Letts. 2010, 22, 287-289.

[5] Chen, Y., Li, H., and Li, M., Sci. Rep. 2012, 2, 622.

[6] Zhang, C., Srinivasan, S., Tang, Y., Heck, M.J.R., Davenport, M.L., and Bowers, J.E., Opt. Exp. 2014, 22, 10202-10209.

[7] Famenini, S., Fonstad, C.G., IEEE Photon. Technol. Lett. 2012, 24, 1849-1851.

[8] Ma. Y., and Tong, L., Front. Optoelectron. 2012, 5, 239-247.

[9] Zimmler, M.A., Capasso, F., Muller, S., and Ronning, C., Semicond. Sci. Technol. 2010, 25, 024001.

[10] Duan, X., Huang, Y., Agarwal, R., and Lieber, C.M., Nature 2002, 421, 241-245

[11] Takiguchi, M., Yokoo, A., Nozaki, K., Birowosuto, M.D., Tateno, K., Zhang, G., Kuramochi, E., Shinya, A and Notomi, M., APL Photonics 2017, 2, 046106.

[12] Malheiros-Silveira, G.N., Lu, F., Bhattacharya, I., Tran, T.-T. D., Sun, H., and ChangHasnain, C.J., ACS Photonics 2017, 4, 1021.

[13] Kim, H., Lee, W.-J., Farrell, A.C., Morales, J.S.D., Senanayake, P., Prikhodko, S.V., Ochalski, T.J., and Huffaker, D.L., Nano Letts. 2017, 17, 3465.

[14] Chen, B., Wu, H., Xin, C., Dai, D., and Tong, L., Nat. Commun. 2017, 8, 20. 
[15] Mouradian, S.L., Schröder, T., Poitras, C.B., Li, L., Goldstein, J., Chen, E.H., Walsh, M., Cardenas, J., Markham, M.L., Twitchen, D.J., Lipson, M., and Englund, D., Phys. Rev. X 2015, 5,31009 .

[16] Sergent, S., Takiguchi M., Tsuchizawa T., Yokoo, A., Taniyama, H., Kuramochi, E., and Notomi, M., ACS Photonics 2017, 4, 1040.

[17] Pauzauskie, P.J., Radenovic, A., Trepagnier, E., Shroff, H., Yang, P., Liphardt, J., Nat. Materials 2006, 5, 97-101.

[18] Hur, S.-H., Park O.O., and Rogers, J.A., Appl. Phys. Letts. 2005, 86, 243502.

[19] Birowosuto, M.D., Yokoo, A., Zhang, G., Tateno, K., Kuramochi, E., Taniyama, H., Takiguchi, M., and Notomi, M., Nat. Mater. 2014, 13, 279-285.

[20] Huang, Y.; Duan, X.; Wei, Q.; Lieber, C. M. Science 2001, 291, 630-633.

[21] Duan, X.; Huang, Y.; Cui, Y.; Wangm J.; Lieber, C.M., 2001, Nature 409, 66-69.

[22] Jin, S.; Whang, D.; McAlpine, M.C.; Friedman, R.S.; Wu, Y.; Lieber, C.M.; Nano Letts. 2004, 4, 915-919.

[23] Lee, C.H.; Kim, D.R.; Zheng, X., PNAS 2010, 107, 9950-9955.

[24] Javey, A.; Nam, S.; Friedman, R.S.; Yan, H.; Lieber, C.M., Nano Letts. 2007, 7, 773-777.

[25] Fan, Z.; Ho, J.C.; Jacobson, Z.A.; Yerushalmi, R.; Alley, R.L.; Razavi, H.; Javey, A., Nano Letts. 2008, 8, 20-25.

[26] Carlson, A., Bowen, A.M., Huang, Y., Nuzzo, R.G., and Rogers, J.A., Adv. Mater. 2012, 24, 5284-5318. 
[27] Bermúdez-Ureña, E., Tutuncuoglu, G., Cuerda, J., Smith, C.L.C., Bravo-Abad, J., Bozhevolnyi S.I., Fontcuberta i Morral A., García-Vidal, F.J., and Quidant, R., Nano Letts. 2017, $17,747-754$.

[28] Sirbuly, D.J., Law, M., Pauzauskie, P., Yan, H., Maslov, A.V., Knudsen, K., Saykally, R.J., Yang, P., PNAS 2005, 102, 7800-7805.

[29] Guilhabert, B., Hurtado, A., Jevtics, D., Gao, Q, Tan, H.H., Jagadish, C., and Dawson, M.D., ACS Nano 2016, 10, 3951-3958.

[30] Gao, Q., Saxena, D., Wang, F., Fu, L., Mokkapati, S., Guo, Y., Li, L., Wong-Leung, J., Caroff, P., Tan, H.H., and Jagadish, C., Nano Letts. 2014, 14, 5206-5211.

[31] Saxena, D., Wang, F., Gao, Q., Mokkapati, S., Tan, H.H., Jagadish, C., Nano Letts. 2015, $15,5342-5348$

[32] Bamiedakis, N., Chen, J., Penty, R.V., and White, I.H., IEEE Phot. Tech. Letts. 2014, 26, 2004.

[33] Trindade, A.J., Guilhabert, G., Massoubre, D., Zhu, D., Laurand, N., Gu, E., Watson, I.M., Humphreys, C.J., and Dawson, M.D., Appl. Phys. Lett. 2013, 103, 253302.

[34] http://www.schott.com/advanced_optics/english/products/optical-materials/thin-glass/thinglass-af-32-eco/index.html

[35] Roelkens, G., Dumon, P., Bogaerts, W., van Thourhout, D., and Baets, R., IEEE Phot. Tech. Letts. 2005, 17, 2613.

[36] Lindenmann, N., Balthasar, G., Hillerkuss, D., Schmogrow, R., Jordan, M., Leuthold, J., Freude, W., and Koos, C., Opt. Exp. 2012, 20, 17667. 

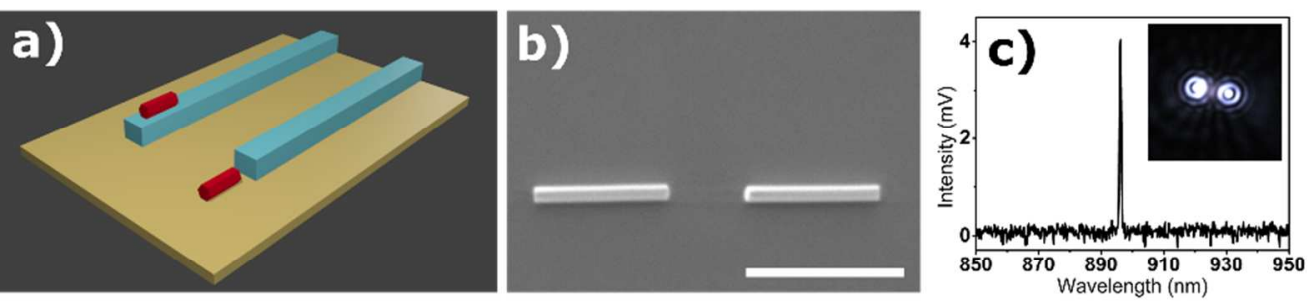

d)

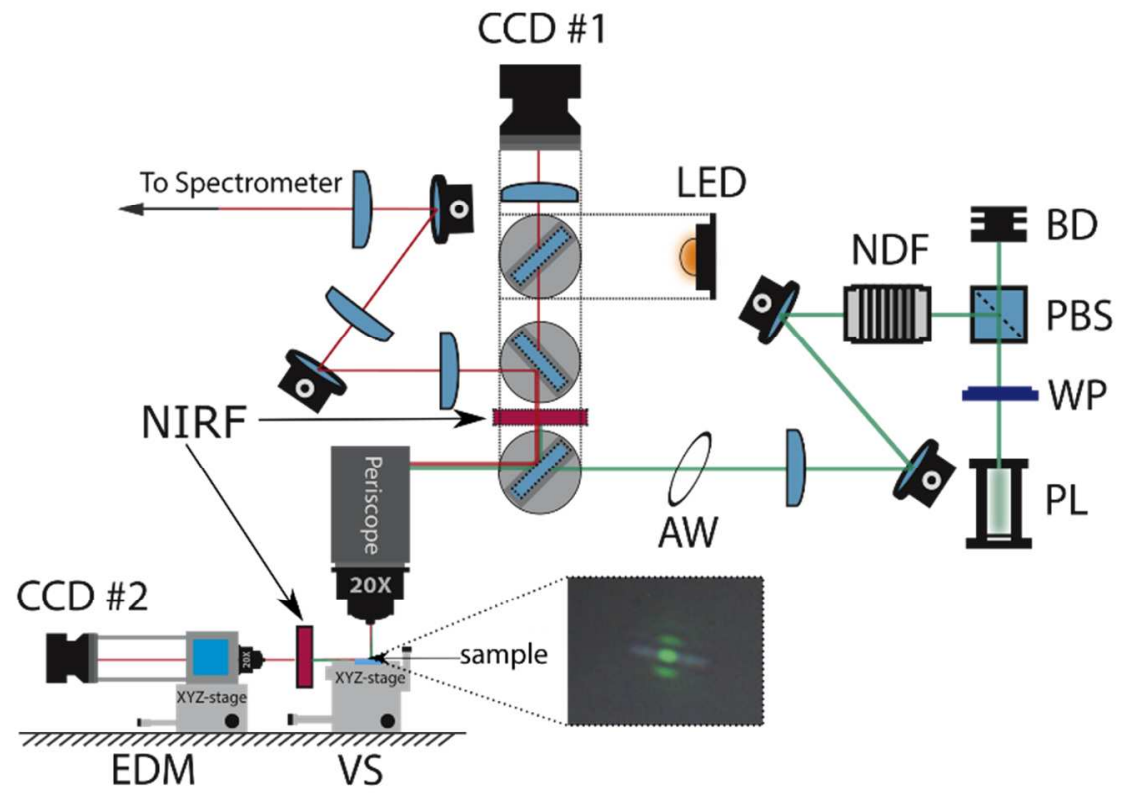

Figure 1. (a) Schematic diagram of the lateral and facet coupling configurations for the integration of NW lasers with waveguides. (b) SEM image of two InP NWs printed in an end-toend configuration for alignment estimation (scale bar: $5 \mu \mathrm{m}$ ). (c) Spectrum and micrograph of an InP NW laser on a silica substrate. (d) Diagram of the $\mu$-photoluminescence setup. PL $=$ pump laser; $\mathrm{WP}=\lambda / 2$ waveplate; $\mathrm{PBS}=$ polarized beam splitter; $\mathrm{BD}=$ beam dump; $\mathrm{NDF}=$ neutral density filter; $\mathrm{AW}=$ attenuation wheel; $\mathrm{NIRF}=$ near-infrared filter; $\mathrm{LED}=$ light emitting diode; $\mathrm{EDM}=$ edge detection module; $\mathrm{VS}=$ vertical setup. 

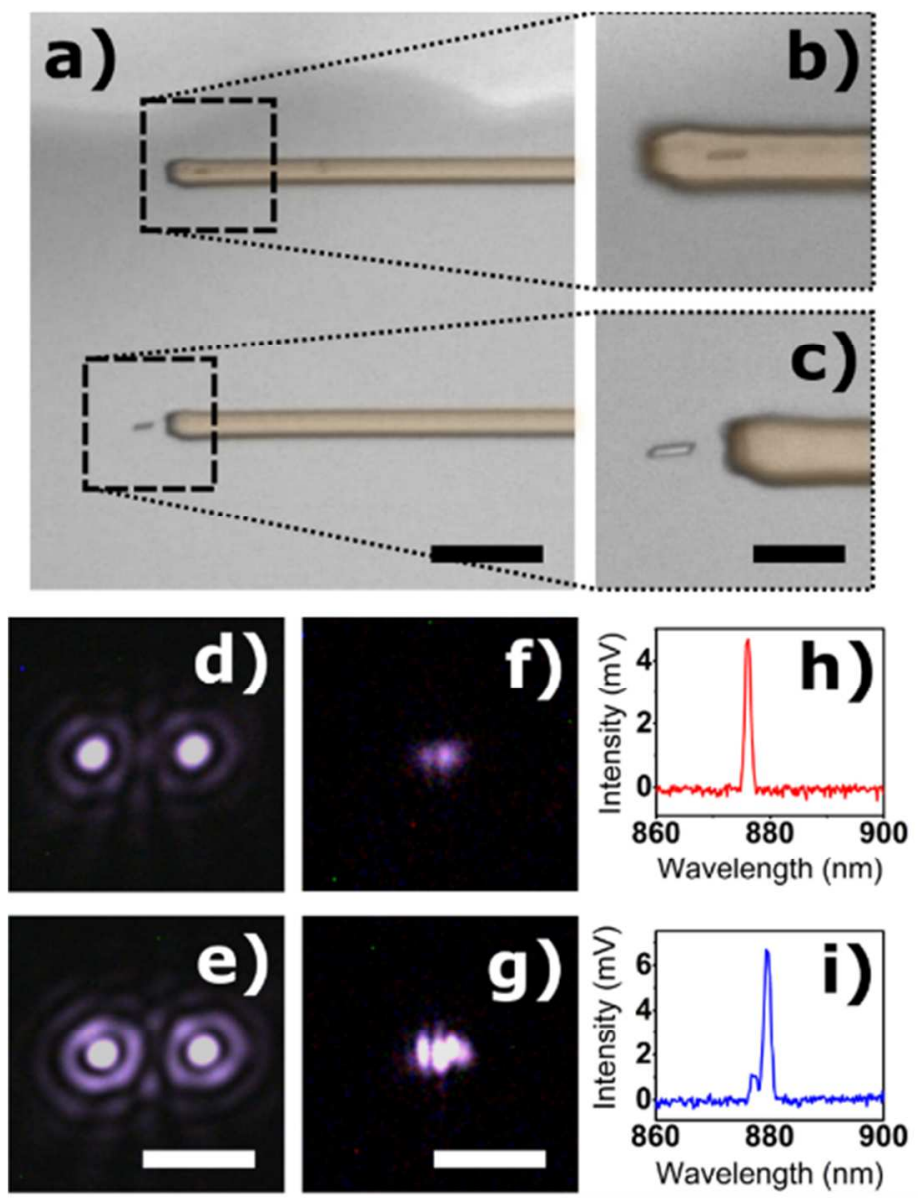

Figure 2. (a-c) Images of two SU-8 waveguides on flexible glass with integrated NW lasers in lateral (up) and facet (bottom) configurations. Scale bar: $30 \mu \mathrm{m}$. (b \& c) Insets for the boxed areas in (a). Scale bar: $10 \mu \mathrm{m}$. (d \& e) Lasing emission (collected in VS) from the NWs coupled in lateral and facet configurations. Scale bar: $7 \mu \mathrm{m}$. (f \& g) Waveguide facet image for the lateral and facet coupled devices. Scale bar: $15 \mu \mathrm{m}$. (h \& i) Spectra for the two integrated NWs in lateral and facet coupling arrangements. 

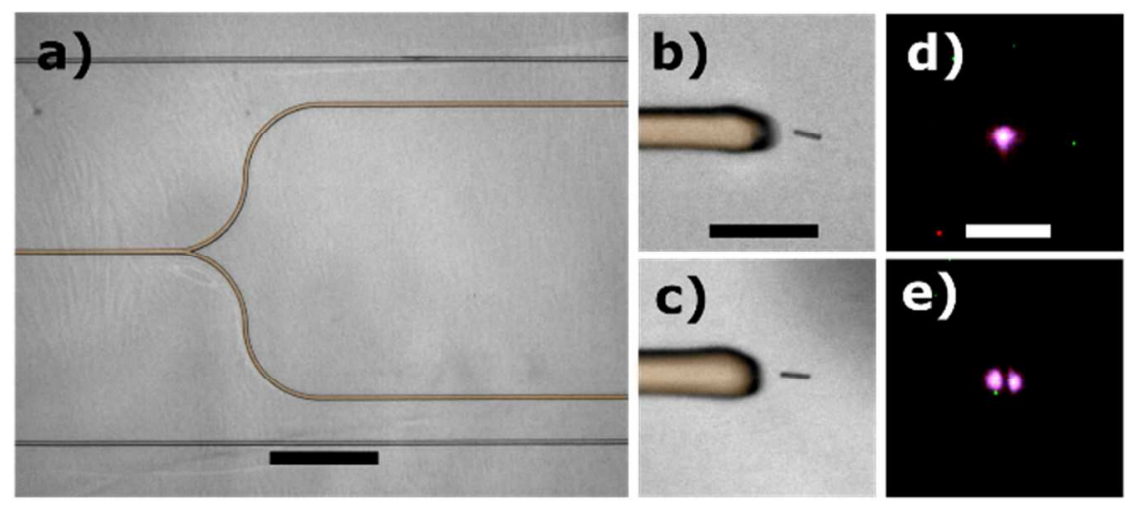

Figure 3. (a) Image of a 2x1 SU-8 Y-junction waveguide. Scale bar: $80 \mu \mathrm{m}$. (b \& c) show facet coupled NW lasers into the 2-input side of the Y-Junction, scale bar: $20 \mu \mathrm{m}$. (d \& e) Waveguide facet images showing the light from the NWs in (b \& c) collected at the 1-output side of the YJunction. Scale bars in (d-e): $10 \mu \mathrm{m}$. 

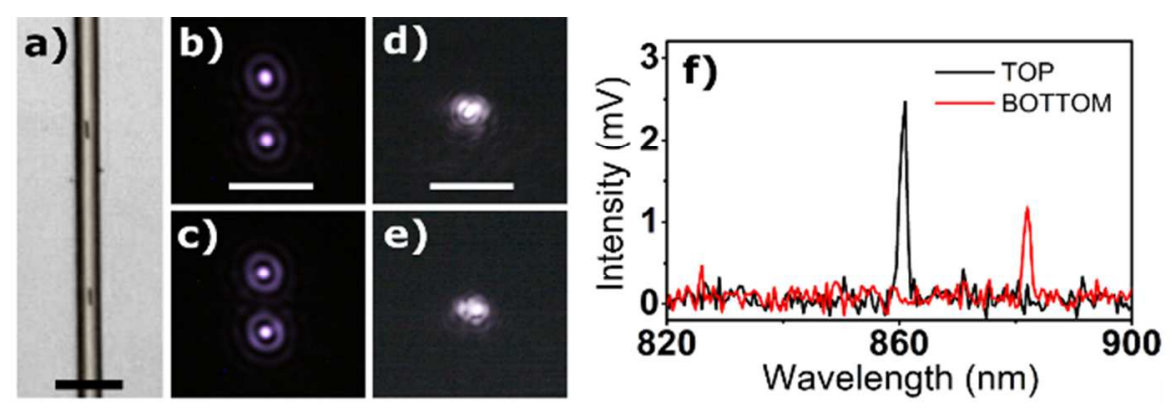

Figure 4. (a) Two InP NW lasers integrated with a waveguide in lateral coupled configuration. Scale bar: $15 \mu \mathrm{m}$. (b \& c) VS collected micrographs of the lasing NWs at the top and bottom parts of the waveguide in (a). Scale bar: $7 \mu \mathrm{m}$. (d \& e) Light collected at the waveguide facet for the NWs integrated at its top (d) and bottom (e) parts. Scale bar: $15 \mu \mathrm{m}$. (f) Individually captured spectra at the waveguide facet for the two integrated NW lasers. 

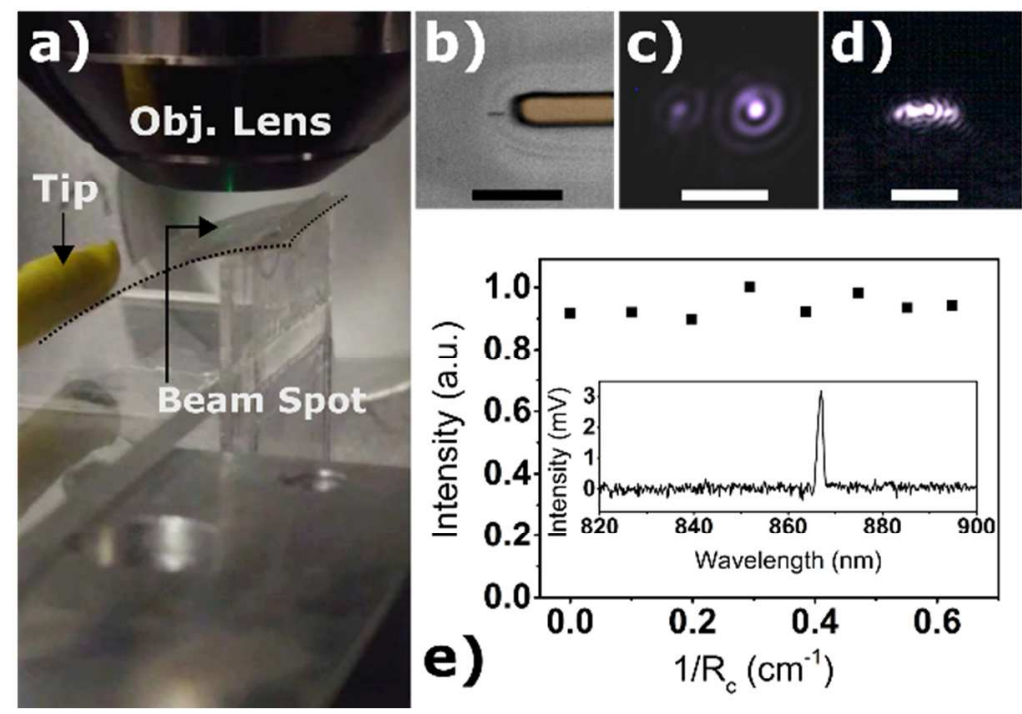

Figure 5. (a) Apparatus used to investigate the effects of substrate bending. (b) NW laser integrated in facet arrangement with a SU-8 polymer waveguide on a flexible glass substrate. Scale bar: $15 \mu \mathrm{m}$. (c \& d) Images of the lasing NW and light collected at the waveguide facet with the substrate bent with a radius of curvature of approx. $1.6 \mathrm{~cm}$. Scale bar: $7 \mu \mathrm{m}$. (e) Normalized light intensity at the waveguide facet as a function of $1 / R_{c}$. Inset shows captured spectral emission of the NW at $1.6 \mathrm{~cm}$ radius of curvature. 

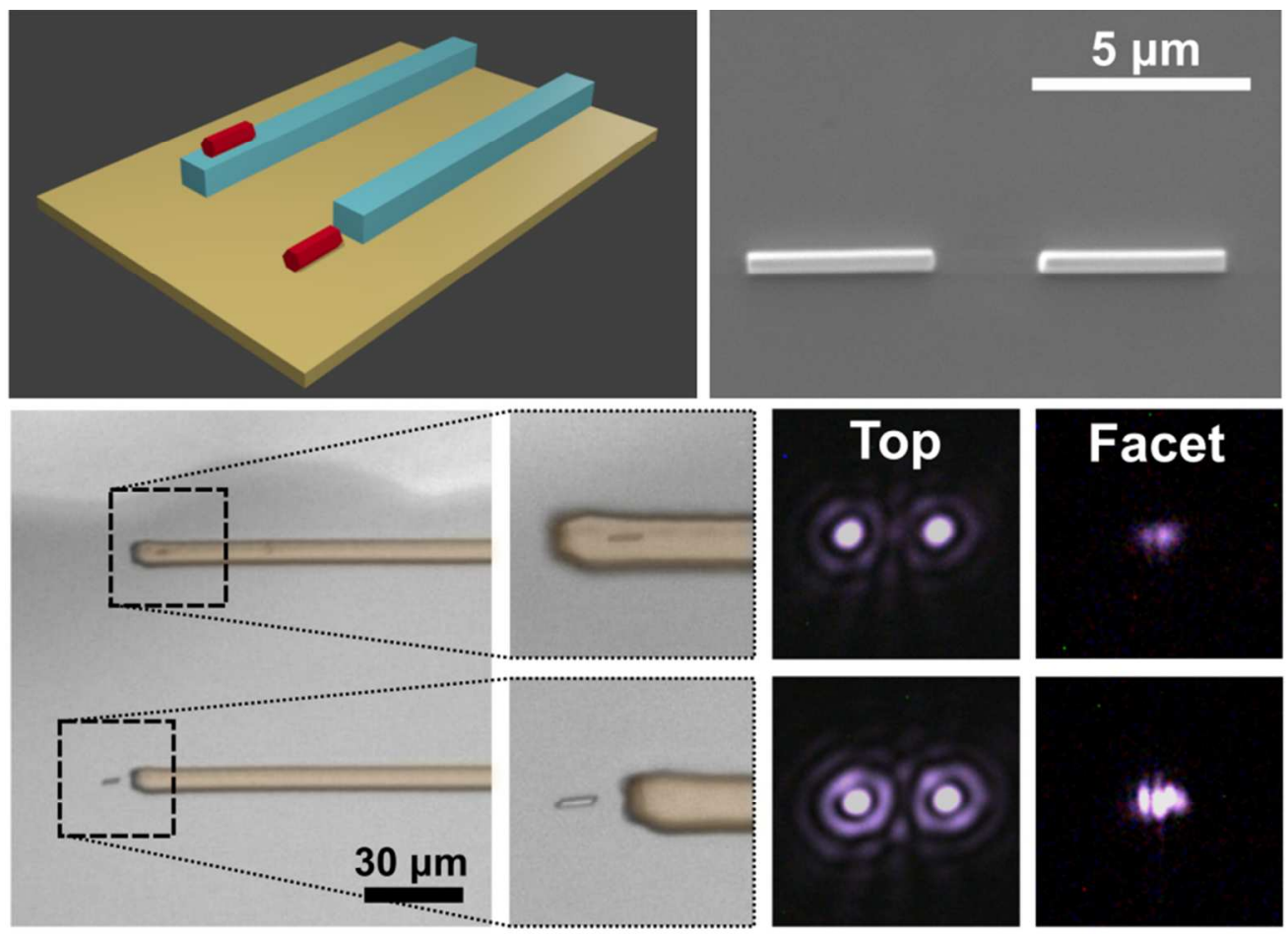

$77 \times 55 \mathrm{~mm}(300 \times 300$ DPI $)$ 